\title{
Aurora-B knockdown inhibits osteosarcoma metastasis by inducing autophagy via the mTOR/ULK1 pathway
}

\author{
Xin Wu ${ }^{1,2+}$, Jia-ming Liu ${ }^{1,2+}$, Hong-hai Song ${ }^{1,2}$, Qi-kun Yang ${ }^{1}$, Hui Ying ${ }^{1}$, Wei-lai Tong ${ }^{1,2}$, Yang Zhou ${ }^{1}$
} and Zhi-li Liu ${ }^{1,2^{*}}$ (1)

\begin{abstract}
Background: Autophagy plays an essential role in metastasis of malignancies. Although our studies showed that Aurora-B facilitate pulmonary metastasis in OS, the mechanism of Aurora-B kinase on autophagy and metastasis in OS has not been explored.

Methods: Clinical-pathological parameters and follow-up information was collected in OS patients. Immunohistochemical staining was performed to detect Aurora-B and LC3 protein in OS tissues. Short hairpin RNA transfection was used to silence Aurora-B in OS cells. Real-time quantitative PCR (RT-qPCR) was performed to detect Aurora-B mRNA expression in OS cells. Aurora-B and autophagy related protein were measured by Western blot. Transmission electron microscopy and laser scanning confocal microscopy were performed to observe the formation of autophagosomes and autolysosomes. Migratory and invasive ability of OS cells were measured by Wound healing and transwell assays. Orthotopic xenograft model was used to evaluate the effect of autophagy mediated by Aurora-B inhibition on pulmonary metastasis of OS.
\end{abstract}

Results: The elevated expression of Aurora-B protein in OS tissues negatively associated with the overall survival of OS patients. Further investigation has found that Aurora-B expression was negatively correlative with autophagy related protein LC3 in OS patient tissues. Knockdown Aurora-B stimulates autophagy and inhibits migratory and invasive ability of OS cells. Mechanistically, Aurora-B knockdown suppressed the mTOR/ULK1 signaling pathway and reactivation of the mTOR/ULK1 pathway decreased autophagy level. Furthermore, the inhibition effect of silencing Aurora-B on migration and invasion of OS was reversed by chloroquine and mTOR activator in vitro and vivo.

Conclusions: Our results suggest that silencing of Aurora-B stimulate autophagy via decreasing mTOR/ULK1 and result in inhibiting OS metastasis. Targeted Aurora-B/mTOR/ULK1 pathway may be a promising treatment strategy for OS patients.

Keywords: Osteosarcoma, Aurora-B, The mTOR/ULK1 pathway, Autophagy, Metastasis

*Correspondence: zgm7977@163.com

${ }^{\dagger}$ Xin Wu and Jia-ming Liu contributed equally to this work

${ }^{1}$ Department of Orthopedic Surgery, The First Affiliated Hospital

of Nanchang University, No.17 Yong Wai Zheng Street, Donghu District, Nanchang, Jiangxi 330006, People's Republic of China

Full list of author information is available at the end of the article

\section{Background}

Osteosarcoma (OS), a highly aggressive tumor with a tendency to metastasize to the lung, is the most common malignant bone tumor in children and adolescents $[1,2]$, and metastasis to the lung is the leading cause of death in patients with OS [3]. Despite the combination of surgery resection with neoadjuvant chemotherapy 
strategies for OS, the 5 year overall survival rate has remained unchanged, at $65-70 \%$, over the past few decades $[4,5]$. In addition, the 5 year survival for metastatic disease is around 20\%, highlighting the need for novel therapeutic targets. Therefore, elucidation of the molecular and cellular drivers involved in the pathogenesis of OS should facilitate the development of effective new strategies for the management of this malignancy.

Autophagy is an intracellular degradation process that removes and recycles damaged proteins and organelles to extend cell longevity. Numerous studies have shown that autophagy is exploited by tumor cells as a highly dynamic process to suppress initial stage of tumor in carcinogenesis by limiting chromosomal instability, restricting oxidative stress, and preventing intratumoral necrosis and local inflammation $[6,7]$. Furthermore, in the early stages of cancer metastasis, autophagy may restrict neoplasm metastasis by suppressing tumor necrosis and inflammatory cell infiltration, and by reducing tumor-induced senescence. Studies have indicated that the induction of autophagy inhibits proliferation, invasion, and migration in bladder cancer and OS cells [8-10]. However, the underlying molecular regulatory mechanisms require further elucidation.

Aurora-B kinase, a member of the aurora kinase family, is a ubiquitously expressed serine/threonine kinase that phosphorylates histone $\mathrm{H} 3$ on Ser10 and variant centrosome protein A on Ser7 in early G2, resulting in the condensation of chromatin 18. Aurora-B participates in the regulation of the spindle assembly complex, chromosome segregation, and cytokinesis [11]. Therefore, silencing or loss of Aurora-B leads to defective chromosome segregation and polyploidy. Abundant evidence has shown that Aurora- $\mathrm{B}$ is expressed at high levels in various malignant tumors, and represents an important antitumor target [12-14]. Amplification or increased expression of Aurora-B has been shown to be associated with poor prognosis in various human malignant tumors [15-17]. Further, Aurora-B is a therapeutic target in non-small cell lung cancer refractory to anti-EGFR therapy [18].Our previous study indicated that the expression of Aurora-B is elevated in OS tissues and cell lines, and that silencing of Aurora-B inhibited the malignant phenotype of OS cells in vitro [19]. However, the mechanisms by which Aurora-B promotes OS pulmonary metastasis have not been fully elucidated to date.

In this study, we investigated the role, and potential underlying mechanisms, of Aurora-B in the pathogenesis of OS, and found that the expression of this kinase is negatively correlated with prognosis and autophagy in OS, which had not been shown before. Furthermore, Aurora-B silencing was shown to inhibit migration and invasion of OS cells by increasing the levels of autophagy mediated by mTOR inhibition. Our results identified a novel as a potential therapeutic target and prognostic biomarker in OS patients.

\section{Material and methods}

Tissues specimens and patients

Sixty-nine OS tissue samples were obtained by surgical biopsy from the First Affiliated Hospital of Nanchang University, China. Patients who had received radiotherapy or chemotherapy before undergoing biopsy were excluded. Donors included 29 females and 40 males, with a mean age of 26 years (range 5-72 years). All OS tissues were subject to pathological examination, and the expression of Aurora-B and LC3 was evaluated through IHC analysis. The clinical parameters are shown in Table 1; follow-up information was missing for 10 of these patients. Informed study protocols were completed in accordance with the declaration of Helsinki and "Guiding Opinions on the Treatment of Animals" in China. The medical ethics committee of the First Affiliated Hospital of Nanchang University has approved this experimental protocol (Jiangxi, China; NO. Y2019-126).

\section{Histology and IHC}

OS tissue samples were fixed in $4 \%$ paraformaldehyde for $20 \mathrm{~min}$, embedded in paraffin, and sectioned to a

Table 1 Correlation of Aurora-B protein expression levels in OS tissues with clinical pathologic parameters

\begin{tabular}{|c|c|c|c|c|}
\hline \multirow[t]{2}{*}{ Variables } & \multirow[t]{2}{*}{ All cases } & \multicolumn{2}{|c|}{ Aurora-B expression } & \multirow[t]{2}{*}{$P$ value } \\
\hline & & Low & High & \\
\hline \multicolumn{5}{|l|}{ Gender } \\
\hline Male & 40 & $19(47.5 \%)$ & $21(52.5 \%)$ & 0.328 \\
\hline Female & 29 & $18(62.1 \%)$ & $11(37.9 \%)$ & \\
\hline \multicolumn{5}{|l|}{ Age } \\
\hline$\leq 20$ & 33 & $19(57.6 \%)$ & $14(42.4 \%)$ & 0.631 \\
\hline$>20$ & 36 & $18(50 \%)$ & $18(50 \%)$ & \\
\hline \multicolumn{5}{|l|}{ Location } \\
\hline Femur/Tibia & 53 & 27 (50.9\%) & $26(49.1 \%)$ & 0.569 \\
\hline Elsewhere & 16 & $10(62.5 \%)$ & $6(37.5 \%)$ & \\
\hline \multicolumn{5}{|c|}{ Tumor size (cm) } \\
\hline$\leq 5$ & 23 & $16(69.6 \%)$ & $7(30.4 \%)$ & 0.076 \\
\hline$>5$ & 46 & $21(45.7 \%)$ & $25(54.3 \%)$ & \\
\hline \multicolumn{5}{|c|}{ Enneking staging } \\
\hline$I+\| A$ & 40 & $26(65 \%)$ & $14(35 \%)$ & $0.031^{*}$ \\
\hline$\|B+1\|$ & 29 & $11(37.9 \%)$ & $18(62.1 \%)$ & \\
\hline \multicolumn{5}{|c|}{ LC3B expression } \\
\hline Low & 34 & $13(38.2 \%)$ & $21(61.8 \%)$ & $0.016^{*}$ \\
\hline High & 35 & $24(68.6 \%)$ & $11(31.4 \%)$ & \\
\hline
\end{tabular}

$P$ value was calculated by Pearson's Chi-Square test. ${ }^{*} P<0.05$ 
thickness of $\sim 3 \mu \mathrm{m}$. These slides were deparaffinized and rehydrated, and then treated with $0.2 \%$ Triton X-100PBS for $10 \mathrm{~min}$ and blocked with $3 \%$ hydrogen peroxide at room temperature for $20 \mathrm{~min}$. These slides were autoclaved in $10 \mathrm{mM}$ citric acid solution to enhance the antigen retrieval for $2 \mathrm{~min}$. The samples were incubated with anti-Aurora-B (Abcam ab45145) and anti-LC3 (Cell signaling technology 2775) overnight at $4{ }^{\circ} \mathrm{C}$. Samples were incubated with appropriate secondary antibodies for 30 min using Histostain Plus kits (Invitrogen, CA, USA). Pictures were captured with the microscope and determined by two pathologists blinded to the specimens.

\section{Cell culture}

HOS and 143B cell lines were obtained from the Type Culture Collection of the Chinese Academy of Sciences, Shanghai, China. All these cells were cultured in DMEM medium (Gibco) supplemented with $10 \%$ fetal bovine serum (FBS, Gibco, 10099141C) with penicillin $100 \mathrm{U} /$ $\mathrm{mL}$ and streptomycin $100 \mathrm{~g} / \mathrm{mL}$ (Solarbio, Shanghai, China). All these cells were cultured at $37{ }^{\circ} \mathrm{C}$ with $5 \%$ $\mathrm{CO} 2$.

\section{Generation of AuroraB knockdown 143B and HOS cells}

143B and HOS cells (1X104) were cultured in $35 \mathrm{~mm}$ cellculture dish and were infected with 2X105 LentivirusVector $(\mathrm{MOI}=20)$ with Aurora-B $(\mathrm{Lv}$-shAuroraB were inserted into lentivirus vector GV115 (GeneChem Co., Ltd., Shanghai, China), 5'-CCG GCTCCAAACTGCTCA GGCATAACTCGAGTTATGCCTGAGCAGTTTGGA GTTTTTG-3 ${ }^{\prime}$ ) for $72 \mathrm{~h}$ and puromycin were incubated to the cells for screening. The efficacy of gene knockdown was determined by Western blot and qRT-PCR.

\section{Western blot analysis}

Human osteosarcoma cells were lysed in RIPA buffer containing protease inhibitor (cocktail and PMSF) for $15 \mathrm{~min}$ on ice. Protein concentrations were measured by BCA Protein Assay kit (Thermo Fisher Scientific). Total cell lysates were electrophoresed by sodium dodecyl sulfate-polyacrylamide gel electrophoresis (SDS-PAGE) on $8 \%-15 \%$ gels and transferred onto PVDF membranes (Millipore). The membranes were blocked with 5\% skim milk (BD) for $60 \mathrm{~min}$ at room temperature and incubated with primary antibodies overnight at $4{ }^{\circ} \mathrm{C}$. Membranes were washed with 1X TBST 3 times and followed by incubation with secondary antibodies for $2 \mathrm{~h}$. The immune complexes were visualized and measured with an ECL system (Bio-Rad, CA, USA) and the digital gel image analysis system (TANON). The primary antibodies included rabbit anti-human AuroraB (Abcam ab45145), anti- $\beta$-tubulin (Abcam ab179513), anti-SQSTM1/P62 (Cell signaling technology 5114), rabbit anti-LC3B (Cell signaling technology 2775), anti-p-mTOR(ser2448)(Cell signaling technology 2971), anti-mTOR(Cell signaling technology 2972), anti-AMPK (Cell Signaling Technology, 2532), anti-pAMPK $\alpha$ (Thr172) (Cell Signaling Technology, 2535), anti-ULK1 (Cell Signaling Technology, 8054) and anti-Pulk1 (Ser555) (Cell Signaling Technology, 5869), mouse anti-human GAPDH (Origene TA802519) and anti-MMP2 (Origene TA806846S). The secondary antibodies included HRP-conjugated Affinipure Goat Anti-Mouse IgG $(\mathrm{H}+\mathrm{L})$ (proteintech SA00001-1) and HRP-conjugated Affinipure Goat AntiRabbit IgG (H+L) (proteintech SA00001-2).

\section{Quantitative real-time PCR analysis}

The total RNA was extracted from the osteosarcoma cell samples by Trizol (Solarbio, Shanghai, China), with the Revert Aid First Strand cDNA Synthesis Kit (Thermo fisher, USA) reverse-transcribed into cDNA. Q-PCR reactions were performed by the TaqMan ${ }^{\mathrm{TM}}$ Fast Advanced Master Mix (Thermo fisher, USA), GAPDH was used as a control. The Aurora-B sequence is Forward $\left(5^{\prime} \rightarrow 3^{\prime}\right)$ AGAAGGAGAACTCCTACCCCT, Reverse $\left(5^{\prime} \rightarrow 3^{\prime}\right)$ CGCGTTAAGATGTCGGGTG, GAPHD sequence is (Forward $\left(5^{\prime} \rightarrow 3^{\prime}\right)$ ) CCACCCATGGCAAAT TCCATGGCA, Reverse $\left(5^{\prime} \rightarrow 3^{\prime}\right)$ TCTAGACGGCAG GTCAGGTCCACC.

\section{LC3 fusion assay}

In order to track autophagosomes, HOS and 143B cells were transfected with lentiviral vectors harboring GFPRFP-LC3 (GeneChem Co., Ltd., Shanghai, China) to obtain cells with stable expression of the GFP-RFP-LC3B protein. Then, these cells were treated with Lv-shAurora$\mathrm{B}$ and control groups for the indicated duration. Images were captured by laser scanning confocal microscopy (ZEISS/LSM 800, Germany) to observe the fluorescence spots mark autophagosomes in these cells.

\section{Transmission electron microscopy (TEM)}

In brief, 143B and HOS cells were collected, fixed with $2.5 \%$ glutaraldehyde and encased. Ultrathin $60-80 \mathrm{~nm}$ sections were prepared with an Ultramicrotome (Leica UC7; Germany) and stained with uranyl acetate (15 min) and Reynolds lead citrate $(15 \mathrm{~min})$. The images were captured by a transmission electron microscope (HITACHI HT7700; Japan).

\section{Transwell migration and invasion assay}

The Millipore $8 \mu \mathrm{m}$ 24-well transwell chamber (Millipore) was used in the osteosarcoma cells migration and invasion. Cells $\left(3 \times 10^{4}\right.$ cells per well $)$ were loaded on FBS-free DMEM in the upper chamber of well coated with or without Matrigel $(100 \mu \mathrm{l} ; 1: 20$ dilution; BD 
Biosciences), The lower chambers were filled with $500 \mu \mathrm{l}$ complete medium mixed with $12 \mu \mathrm{m}$ CQ or $2 \mu \mathrm{m}$ MHY1485 and a corresponding dose of PBS as control. After $12 \mathrm{~h}$, the lower chambers mixtures were removed and replaced with $500 \mu \mathrm{l}$ new complete medium. Another $12 \mathrm{~h}$ later, cells on the upper surface of the well were removed, and invasion cells passed through the well on the bottom were stained with $1 \%$ crystal violet. Cells in six randomly selected fields were counted and photographed (magnification 10X).

\section{Wound healing assays}

143B and HOS cells were grown to confluence in 6-well plates in the density of $5-8 \times 10^{6}$ cells per well. When the cells were grown to $90 \%$ confluence, we used $20 \mu \mathrm{l}$ pipette tip to scratch wounds through the center of the plate. The cells were washed three times with PBS to remove the floated cells and then incubated with $1 \%$ FBS MDEM mixed with $12 \mu \mathrm{m}$ CQ or $2 \mu \mathrm{m}$ MHY-1485 and a corresponding dose of PBS as control at $37^{\circ} \mathrm{C}$ for $8 \mathrm{~h}$. The mixtures were removed and replaced by new $1 \%$ FBS MDEM. Images were captured at different time points (0 and $24 \mathrm{~h}$ ), and the migration distance was measured by ImageJ compared with the time zero.

\section{In vivo assay}

All experimental protocols were approved by the Institutional Animal Care and Use Committee of Nanchang University (Jiangxi, China; NO. Y2019-126). We purchased female BALB/C nude mice at 6 weeks of age from the Nanjing Biomedical Research Institute of Nanjing University (NBRI, Nanjing, China); mice were housed in the SPF (Specific Pathogen Free) Transgenic Animal Facility of Nanchang University. The protocol for generation of a spontaneous metastasis/orthotopic osteosarcoma mouse model was applied as reported in our previous study [20]. The mice were randomly divided into four groups (Ctrl group, AZD2811 groups, ADZ2811+3BDO group, and AZD2811+CQ groups; $\mathrm{n}=6$ in each group). Drugs (normal saline, AZD2811 $(150 \mathrm{mg} / \mathrm{kg})$, ADZ2811 (150 mg/kg), and 3BDO $(80 \mathrm{mg} /$ $\mathrm{kg})$ mixture, AZD2811 (150 mg/kg), and CQ $(80 \mathrm{mg} / \mathrm{kg})$ mixture) were separately administered to mice via intraperitoneal injection, twice a week. After 6 weeks, the tumors were dissected and fixed in $10 \%$ formalin. The lung tissues were dissected to evaluate pulmonary metastasis by optical microscope using the Vivo imaging system (Berthold LB983; Germany). The tissues were fixed in $10 \%$ formalin for further detection.

\section{Statistical analysis}

All these quantitative data were presented as the mean $\pm S D$. Student's t-test was performed for two-sample analysis, and one-way ANOVA was performed for multiple-sample analysis by using GraphPad Prism 7 software. $P<0.05$ was considered a statistically significant difference.

\section{Results \\ Aurora-B expression was negatively correlated with prognosis in OS.}

To investigation the relationship of Aurora-B expression with prognosis in OS. We examined the expression of Aurora-B by immunohistochemical staining and collected the follow-up information from OS patients. We found that the expression of Aurora-B protein is closely correlated with Enneking stage (Table 1; Fig. 1a, b) $(P<0.01)$. Moreover, Kaplan-Meier analysis showed that high levels of Aurora- $B$ expression were negative correlated with poor overall survival (Fig. 1c) $(P<0.01)$ and lower metastasis-free survival (Fig. 1d) $(P<0.01)$ in these OS patients. These results collectively indicate that the expression of Aurora-B protein was negatively correlated with prognosis in OS.

\section{Aurora-B knockdown induces autophagy in OS}

To investigate the correlation between Aurora- $B$ and autophagy, we first applied immunohistochemical staining to examine the Aurora-B and LC3 expression in OS tissues. In the tissues with Aurora-B-positive expression, LC3 protein was poorly expressed, and the opposite was observed in the tissue samples that tested negative for Aurora-B expression (Table 1; Fig. 2a, b) $(P<0.05)$. These results suggested that a potential correlation between Aurora-B and autophagy in OS. Further investigate the effect of Aurora-B on autophagy in OS cells, we knocked down endogenous Aurora-B with shRNA (short-hairpin RNA) in 143B and HOS cells by lentiviral infection (Fig. 2c, d). The RFP-GFP-LC3 fusion assay was performed to observe the formation of autophagosomes and autolysosomes in OS cells. The number of yellow LC3 puncta (representing autophagosomes) and red LC3 puncta (representing autolysosomes) were both higher in Aurora-B silenced cells than in cells infected with scrambled lentivirus, which represented the negative control (Fig. 2e). Similar results were observed by transmission electron microscopy (Fig. 2f). Furthermore, the ratio of LC3-II to LC3-I was significantly increased, whereas p62 expression was decreased, in cells with Aurora-B-knockdown relative to that in cells infected with scrambled lentiviruses (Fig. 2g). The results indicate that inhibition of Aurora-B enhanced autophagy in OS cells. Furthermore, to distinguish whether the accumulation of LC3 is due to the induction of autophagy or the depletion of autophagy flux, cells were treated with the specific autophagy inhibitor chloroquine $(\mathrm{CQ})$, which inhibits 


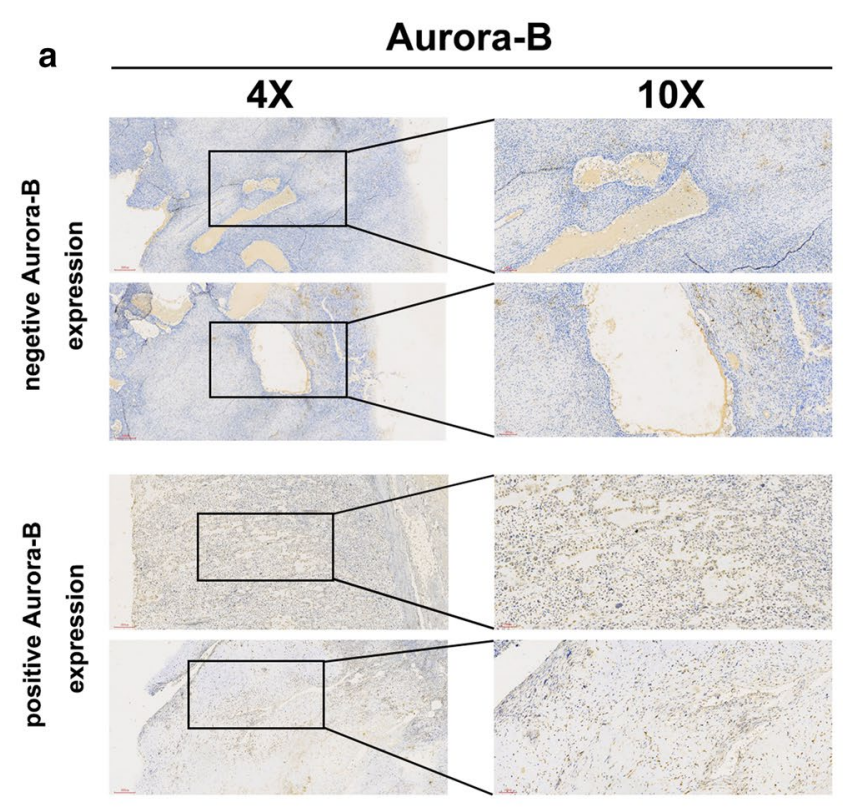

\section{b Enneking stage}
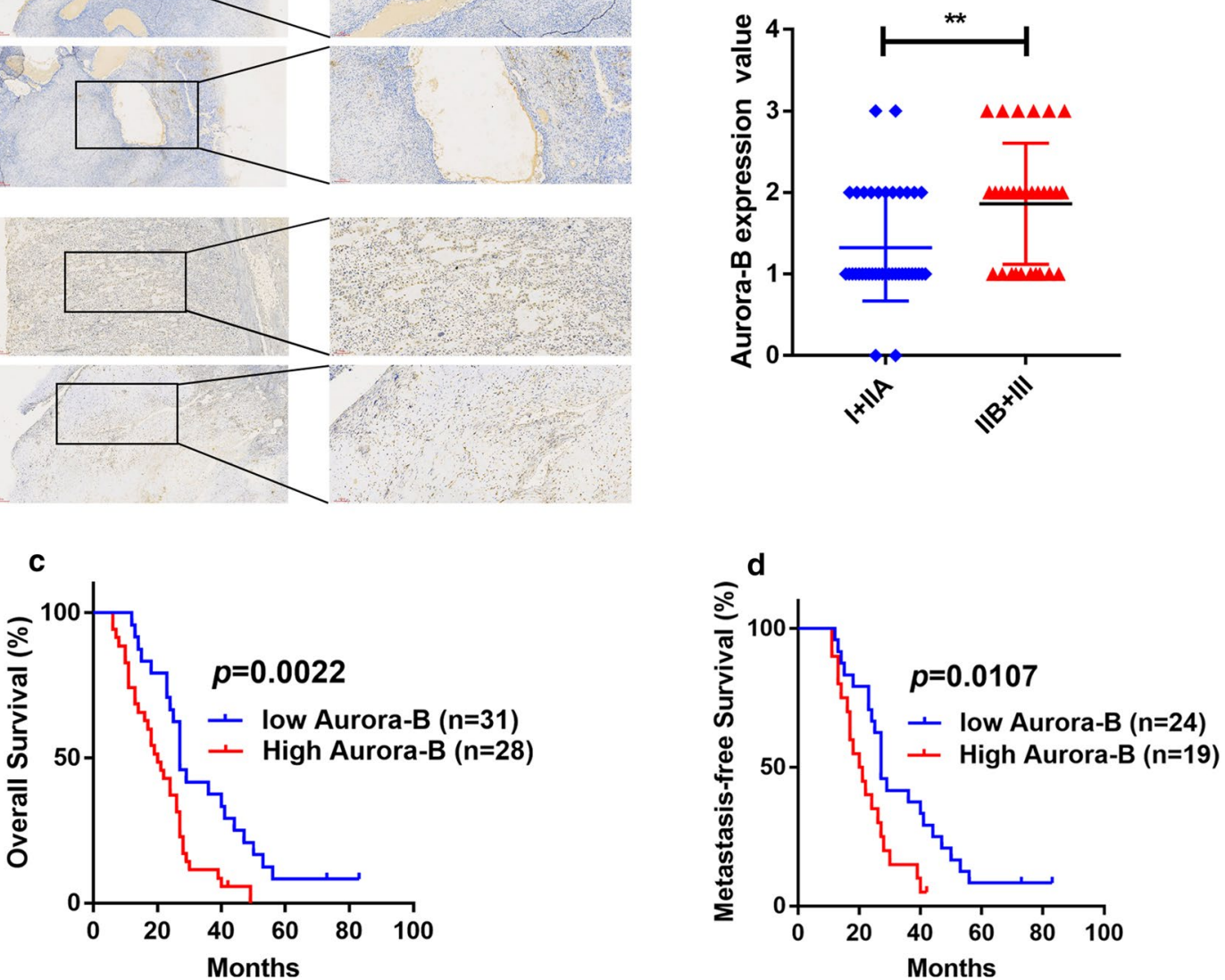

Fig. 1 Aurora-B expression was negatively correlated with prognosis in OS. Tissue samples and data for clinical-pathological parameters were collected from 69 OS patients. The expression of Aurora-B protein in tumor tissues were detected by immunohistochemical staining (a). Statistical analysis of Enneking stage classification (b), ${ }^{* *} P<0.01$ in 69 OS patients, among them, there are 40 cases of I $+\| \mathrm{A}$ and 29 cases of $I I B+I I$. c $P<0.01$, d $P<0.05$ Kaplan-Meier survival analysis according to Aurora-B expression in 59 patients to be followed up

(See figure on next page.)

Fig. 2 Aurora-B knockdown induces autophagy in OS. The expression of LC3 protein in tumor tissues were detected by immunohistochemical staining (a), and analyzed by staining scores was performed in 69 OS patients. There are 37 cases of low Aurora-B expression and 32 cases of high Aurora-B expression. $\mathbf{b} * P<0.05 .143 \mathrm{~B}$ and $\mathrm{HOS}$ cells were infected with shAurora-B and scrambled lentivirus. Aurora-B expression in $143 \mathrm{~B}$ and $\mathrm{HOS}$ cells was knocked down using shAurora-B lentivirus, and analyzed by western blotting (c) and GRT-PCR (d).***P<0.01. The RFP-GFP-LC3 fusion assay was performed and cells were visualized by laser scanning confocal microscopy (e); fusion numbers were quantified by randomly selecting three views per slide. ${ }^{* * *} P<0.01$, Scale bars: $20 \mu \mathrm{m}$. Autophagosomes and autolysosomes were observed with a transmission electron microscope (f). Scale bars: $5 \mu \mathrm{m}$ (left pictures) and $1 \mu \mathrm{m}$ (right pictures). The protein level of LC3-I, LC3-II, and SQSTM1 were analyzed by western blotting (g). Infected cells were treated with $10 \mu \mathrm{M}$ CQ (chloroquine diphosphate) for $24 \mathrm{~h}$. The total level of LC3-I, LC3-II, and SQSTM1 proteins were used to detect the autophagic flux (h)

autophagosome-lysosome fusion, thus preventing LC3 degradation. As shown in Fig. 1f, the LC3 II protein level was significantly increased following treatment with CQ in both control and Aurora-B-silenced cells compared with that in cells that were not treated with CQ (Fig. 2h). These results indicate that Aurora-B knockdown more 
a
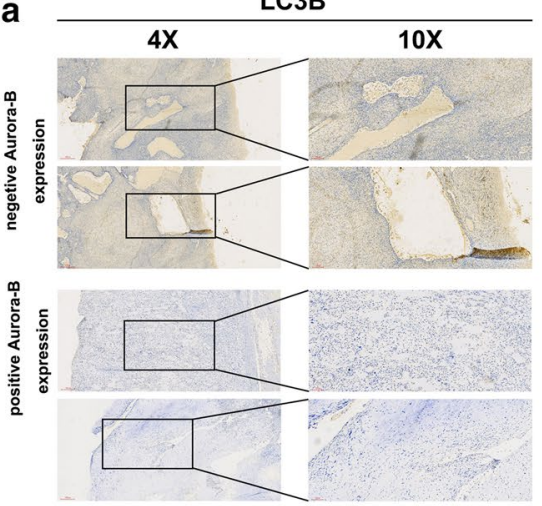

c

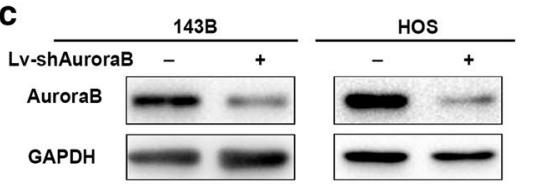

e

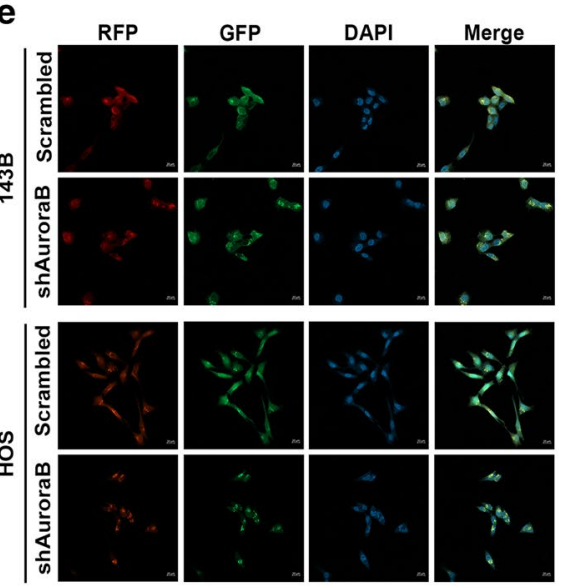

g

LV-shAuroraB

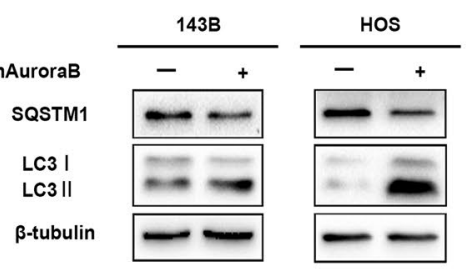

h

b LC3B expression

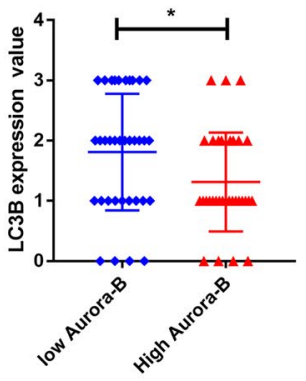

d
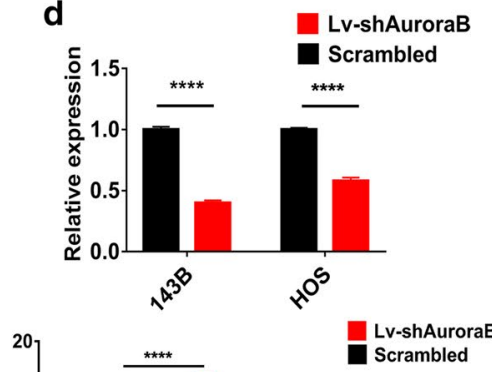

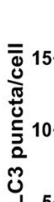

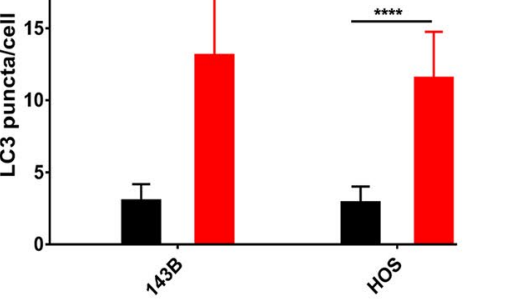

f Scrambled Lv-shAuroraB

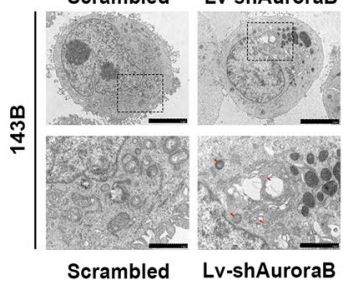

Scrambled Lv-shAuroraB

온

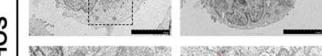

-

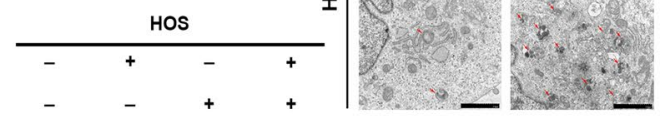

LV-shAuroraB -
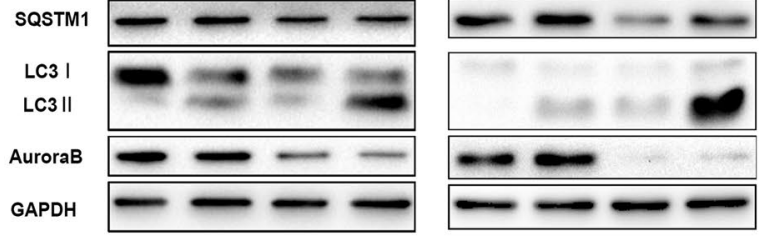
likely enhances autophagic flux than inhibits autophagy in OS cells.

\section{Silencing Aurora-B induces OS cells autophagy by suppressing the mTOR/ULK1 pathway}

The mTOR/ULK1 pathway is known to regulate autophagy, and the inactivation of this pathway has been shown to enhance autophagy levels [21, 22]. AMPK is a critical sensor of stress and energy metabolism that negatively regulates mTOR, activating autophagy initiation factor ULK1 and promoting autophagic flux [23, 24]. To explore the molecular mechanisms involved in the enhancement of autophagy by Aurora-B knockdown, we examined the expression of proteins involved in the mTOR/ULK1 pathway in Aurora-B-knockdown 143B and HOS cells. Interestingly, as shown in Fig. 3a, the phosphorylation levels of AMPK and ULK1 were upregulated and those of mTOR were downregulated in AuroraB knockdown 143B and HOS cells, which was consistent with the observed changes in total AMPK, ULK1, and mTOR levels. These results suggest that Aurora-B knockdown enhances autophagy by decreasing mTOR/ULK1 pathway activity. Recent evidence has additionally demonstrated that the suppression of the malignant phenotype of OS cells by Aurora-B silencing might be achieved via autophagy-mediated activation of the mTOR/ULK1 signaling pathway. Thus, to further investigate the role of the mTOR/ULK1 pathway during Aurora-B silencing-induced autophagy, we investigated the expression of the autophagy-related protein p62 after treatment with MHY-1485, a well-known mTOR activator [25], or not, in Aurora-B-knockdown or scrambled OS cells. In the Aurora-B-knockdown OS cells, MHY-1485 treatment activated mTOR/ULK1 signaling and increased p62 levels. In the scrambled groups, after treatment with MHY-1485, the expression of p62 and phosphorylation of mTOR increased (Fig. 3b). These results reveal that inhibition of Aurora-B induced autophagy in OS cells via decreasing the mTOR/ULK1 pathway.

\section{Aurora-B knockdown-induced autophagy inhibits migration and invasion in OS cells}

Autophagy is known to be involved in tumor progression $[26,27]$. In order to elucidate the effect of Aurora$B$ inhibition induced autophagy on the metastasis of OS cells, wound healing, transwell migration and invasion assays were performed to detect migration and invasion ability of OS cells. We surprisingly found that Aurora-B inhibition significantly decreased the motility and invasive ability of $143 \mathrm{~B}$ and HOS cells. After treatment with $\mathrm{CQ}$, the attenuated ability could be reversed. (Fig. 4a-f). These findings indicate that autophagy plays an essential role in the process of Aurora-B affecting the ability of motility and invasive in OS cells. Autophagy is reported to inhibit cell migration by accelerating the degradation of MMP family proteins [26, 27], therefore, we sought to determine the MMP2 protein levels in Aurora-B-knockdown OS cells with or without CQ treatment. Results showed that the suppression of MMP2 expression due to Aurora-B knockdown could be restored by CQ treatment (Fig. 4g). These findings demonstrate that inhibitio of Aurora B suppresses the migration and invasion of OS cells by stimulating autophagy.

\section{Activation of the mTOR/ULK1 pathway reverses the effect of Aurora-B inhibition on migration and invasion of OS cells}

To confirm whether the mTOR/ULK1 pathway contributes to Aurora-B-induced suppression of the malignant phenotype in OS cells, we performed wound healing, transwell migration, and invasion assays to evaluate the migration and invasion ability of Aurora-B-knockdown OS cells treated with MHY1485 or not. The results revealed that the inhibition of migration and invasion ability induced by Aurora-B knockdown could be reversed by MHY1485 treatment (Fig. 5a-f). And it indicates that activation of the mTOR/ULK1 pathway reverses the induction of Aurora-B knockdown-induced autophagy, which inhibits migration and invasion in OS cells.

\section{Aurora-B knockdown inhibits metastasis via $\mathrm{mTOR}$-mediated autophagy in vivo}

In order to investigate how the inhibition of Aurora-B affects the metastasis of OS, we established an orthotopic xenograft model using the luciferase-labeled 143B cell line (Fig. 6a). Bioluminescent imaging analysis revealed that the number of metastatic foci in the lung was significantly decreased when treated with AZD2811, a specific Aurora-B inhibitor in these mice models. Furthermore, 3BDO, a new mTOR activator, or CQ injection reversed these effects. H\&E staining and lung anatomy confirmed this result (Fig. 6b-d). These results suggested that Aurora-B knockdown inhibits OS metastasis via mTORmediated autophagy in vivo.

\section{Discussion}

Aurora-B, a serine/threonine kinase, plays a vital role in a variety of biological behaviors in cells, such as mitosis and the cell cycle [28]. Recent evidence has demonstrated that Aurora-B accelerates the progression of lung cancer [12], gastric cancer [29], prostate cancer [30], and OS [27]. Previously, we found that Aurora-B positive expression rate is increased in human OS tissues with pulmonary metastasis compared with that in non- metastasis tissues [31]. Here, to further examine the role of 


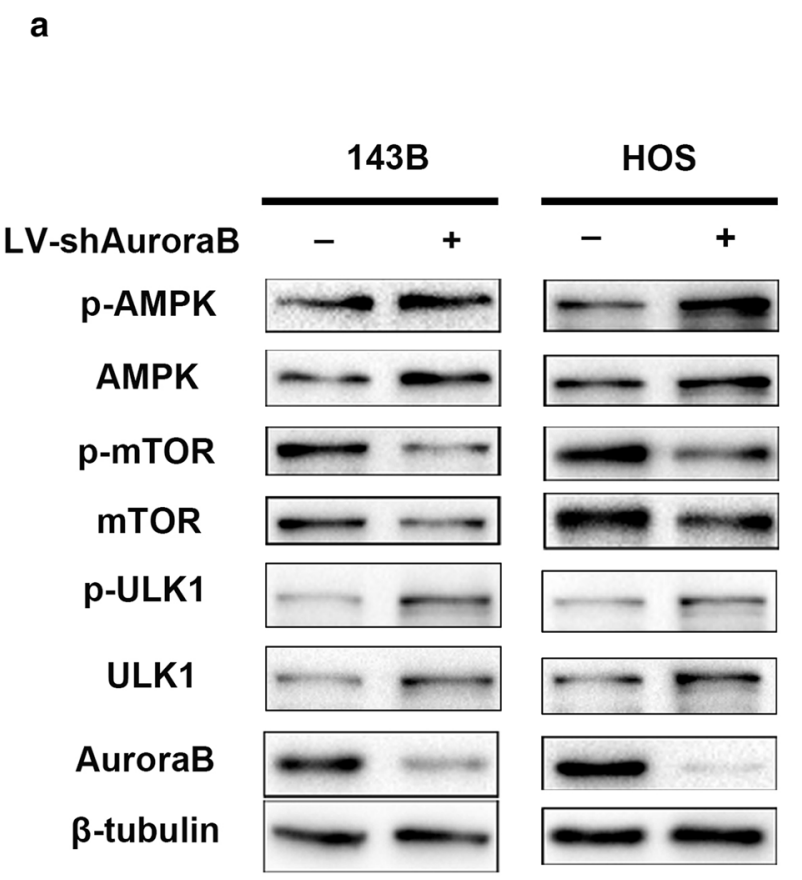

b
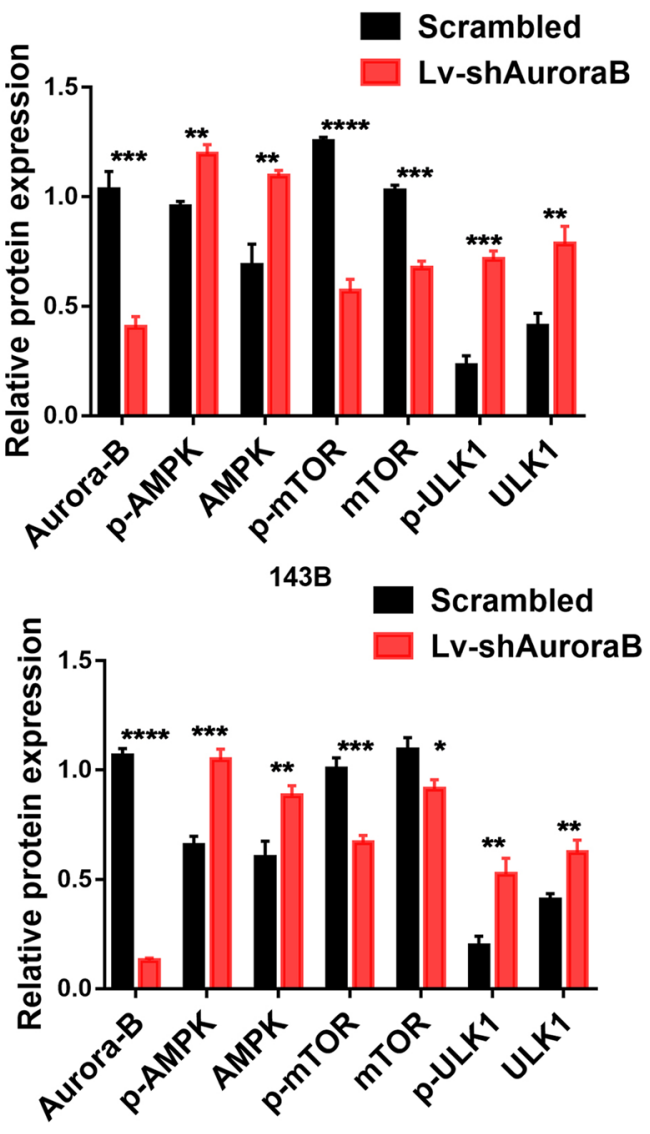

HOS
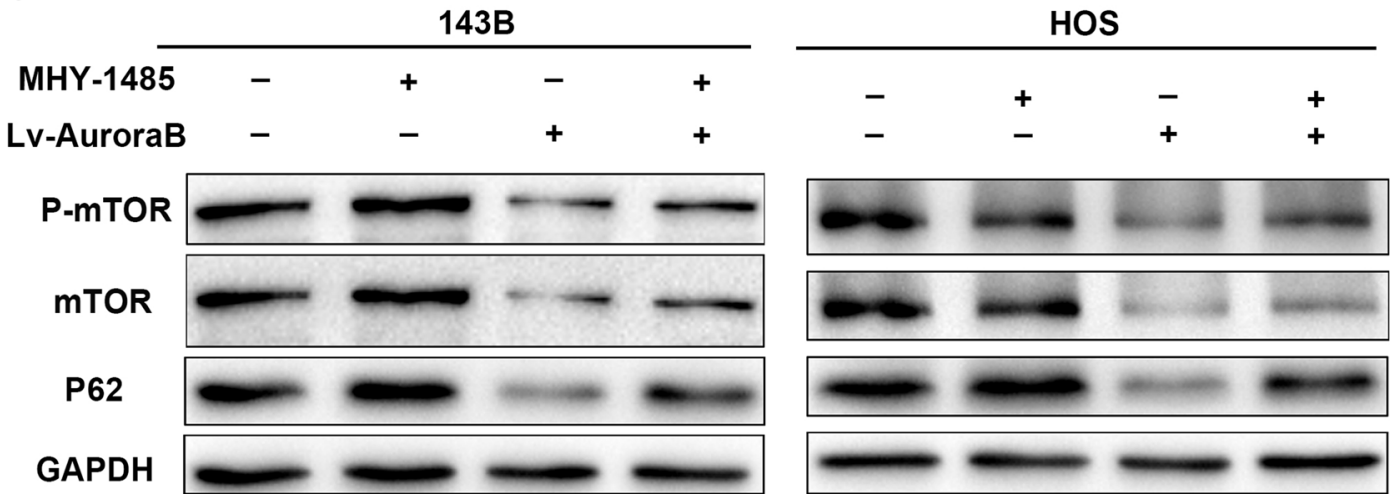

Fig. 3 Silencing Aurora-B induces OS cells autophagy by suppressing the mTOR/ULK1 pathway. Aurora-B silencing and corresponding contrast OS cells were used to measure examine the mTOR/ULK1 pathway signaling and autophagy. The protein levels of AMKP, p-AMPK, mTOR, p-mTOR, ULK1, and p-ULK1 in Aurora-B B-knocked down 143B, and HOS cells were analyzed by western blotting (a). Treated Following treatment of these OS cells with or without MHY-1485 (2 $\mu \mathrm{M}, 3 \mathrm{~h})$ in these OS cells, or not, the expression of P62p62, p-mTOR, and mTOR was detected investigated by western blotting (b)

(See figure on next page.)

Fig. 4 Aurora-B knockdown-induced autophagy inhibits migration and invasion in OS cells. The migration and invasion ability of

Aurora-B-knockdown 143B and HOS cells treated with or without CQ $(12 \mu \mathrm{M}, 12 \mathrm{~h})$ were examined by transwell migration $(\mathbf{a}, \mathbf{b}$ **** $P<0.01$, Scale bars: $100 \mu \mathrm{m})$ and invasion $\left(\mathbf{c}, \mathbf{d}{ }^{*} P<0.05,{ }^{* * *} P<0.01,{ }^{* * *} P<0.01,{ }^{* *} P<0.01\right.$, Scale bars: $\left.100 \mu \mathrm{m}\right)$ assays in vitro. Wound healing assay was performed with scrambled and shAurora-B 143B and HOS cells treated with CQ $(12 \mu \mathrm{M}, 12 \mathrm{~h})$ for $24 \mathrm{~h}$ or not $\left(\mathbf{e}, \mathbf{f}{ }^{* *} P<0.01,{ }^{* * *} P<0.01,{ }^{*} P<0.05\right.$, Scale bars: $100 \mu \mathrm{m})$. MMP2 protein expression of Aurora-B-knockdown cells treated with CQ $(10 \mu \mathrm{M}, 24 \mathrm{~h})$ or not was analyzed by western blotting ( $\mathbf{g})$ 


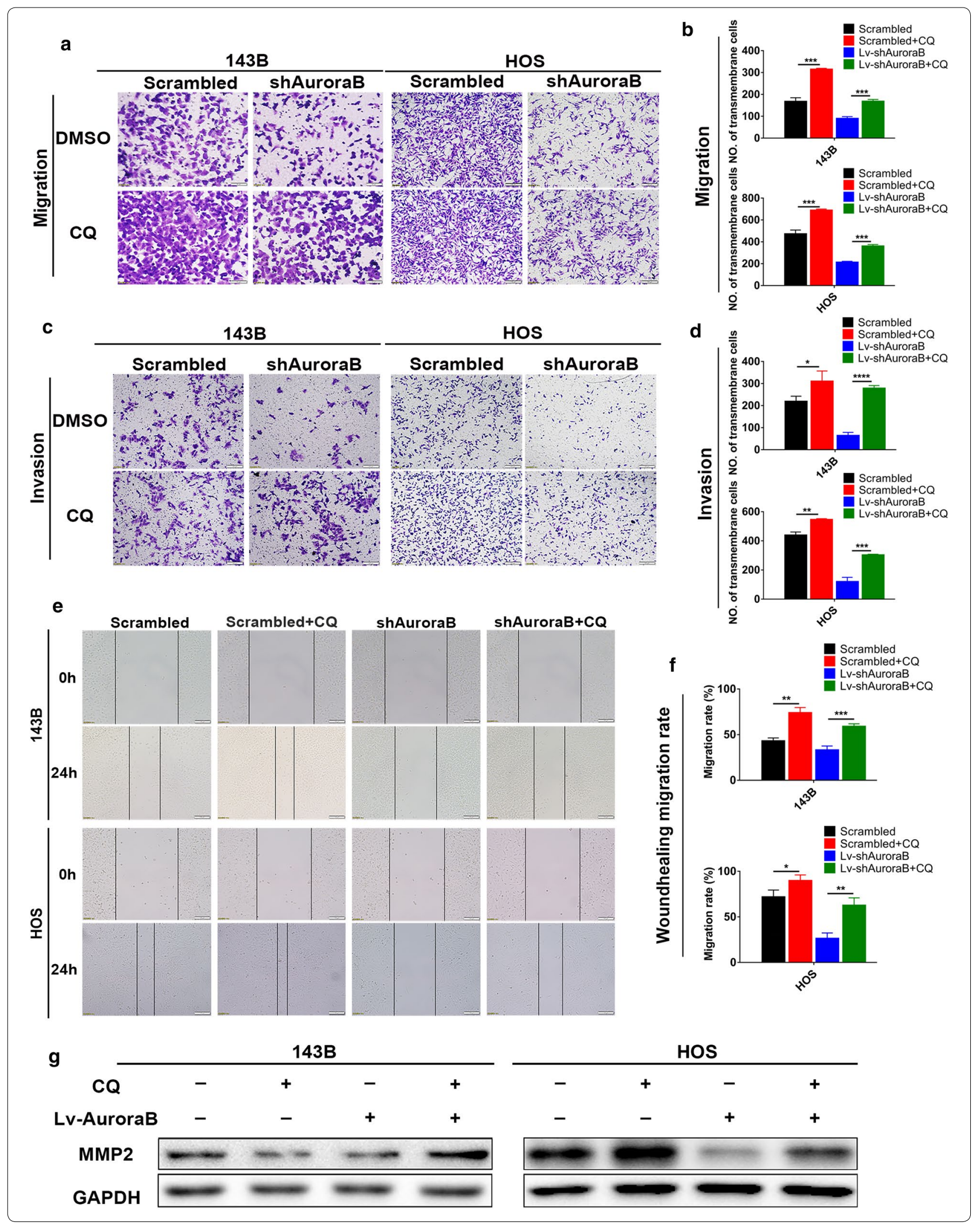




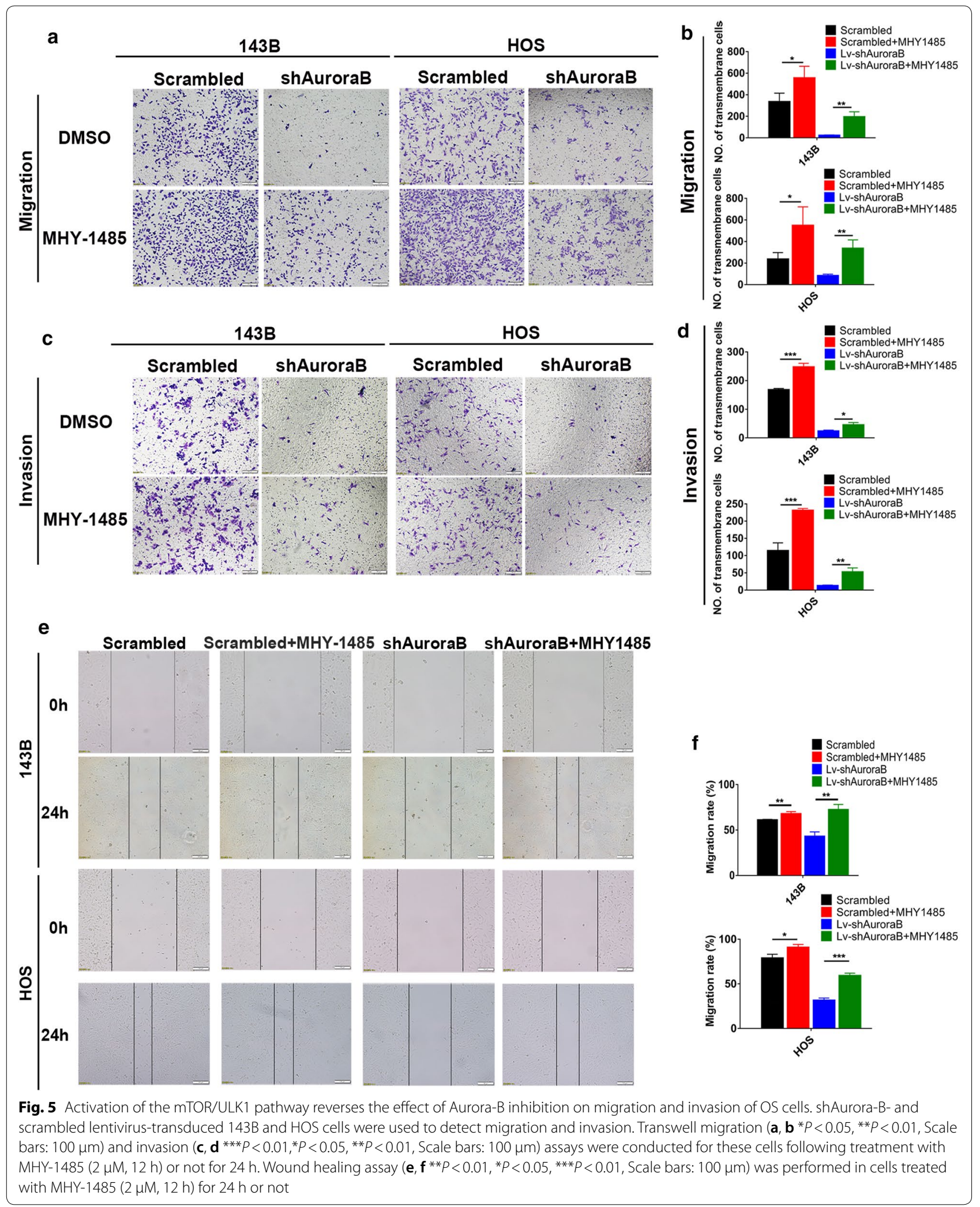


this kinase in OS metastasis and autophagy, we investigated the prognostic value of Aurora-B expression in 59 OS patients, and evaluated this kinase relationship with LC3 protein expression. We demonstrated that patients with high Aurora-B expression are likely to have a poor prognosis and low LC3 expression. In our previous study, Aurora- $\mathrm{B}$ was shown to partly activate migration and invasion via regulation of the NF- $\mathrm{kB}$ pathway and induction of MMP2 expression in OS [32]; however, the mechanism underlying Aurora-B-induced metastasis in OS requires elucidation. Interestingly, in the present study, we initially found that the effect of Aurora-B on OS invasion and metastasis could be regulated by mediating autophagy via the mTOR/ULK1 pathway; these findings had not been reported before and extended those of our previous study and confirmed the notion that Aurora- $\mathrm{B}$ knockdown suppresses autophagy-mediated OS metastasis via the mTOR/ULK1 pathway, and represents a useful biomarker of OS prognosis.

Autophagy, a complex biological behavior, plays a dual role in tumors such as OS [33]. In the early stages of tumorigenesis, autophagy can function as a tumor suppressor by inhibiting chromosomal instability, limiting oxidative stress, and inducing autophagic cell death to hinder metastasis and enhance the efficacy of chemotherapeutic drugs. In the later stages of tumorigenesis, autophagy can also function as a tumor activator by promoting metabolism and anoikis resistance, and maintaining homeostasis in tumor cells to promote metastasis and cancer progression [34]. In the present study, we investigated the potential role of Aurora-B in autophagy regulation, and found that a potential correlation between Aurora-B and autophagy in OS tissues and inhibition of Aurora-B could enhance autophagy in OS cells. Further investigation revealed that the use of chloroquine to inhibit autophagy activation promotes cell migration and invasion. This is consistent with the effect of autophagy on metastasis, as observed by Zhang et al. [35], and Liu et al. [36]. In contrast, Liu et al. found that microRNA-210-5p promotes metastasis by suppressing PIK3R5-induced autophagy via the mTOR pathway [37]. The differences in our conclusions may result from the method of autophagy inhibition: chloroquine suppresses autophagy by blocking autophagosome-lysosome fusion. Nevertheless, it is not an entirely specific autophagy inhibitor [38]. Specific autophagy inhibitors seem to be more suitable for the inhibition of autophagy, e.g. via RNA silencing or gene-specific targeting technologies to knockdown genes encoding autophagy-relevant components (e.g., ATG5, ATG7, or BECN1).

Autophagy is regulated by several signaling pathways, including mTOR, AMPK, and PKA [39-41]. Cao et al. found that the activation of autophagy is regulated by the AMPK/mTOR/ULK1 pathway in triple-negative breast cancer cells [24], whilst Zhang et al. demonstrated that thymoquinone inhibits metastasis in renal cell cancer cells by inducing autophagy via the AMPK/mTOR/ULK1 signaling pathway [42]. Emerging evidence demonstrates that the AMPK/mTOR/ULK1 signaling pathway is a critical regulator of tumor autophagy, and participates in the progressions of numerous tumor types. In this study, we found that the phosphorylation and total levels of AMPK and ULK1 expression were upregulated and those of mTOR were downregulated in Aurora-B-knockdown cell lines 143B and HOS. Further, because mTOR is wellknown to regulate autophagy, we speculated that Aurora$B$ knockdown enhances autophagy by inhibiting the mTOR/ULK1 signaling pathway. In our previous study, we demonstrated that Aurora- $\mathrm{B}$ alters the malignant phenotype of OS cells partly via the PI3K/Akt/NF-k $\beta$ pathway [43], however, the underlying mechanism remains to be understood. Herein, we found that Aurora-B silencing inhibits migration and invasion of OS cells, whereas reactivation of mTOR phosphorylation suppresses Aurora-B knockdown-induced autophagy and reverses the inhibition of migration and invasion in OS cells. A similar phenomenon was observed in vivo. Our study illustrates the role of the mTOR/ULK1 signaling pathway in Aurora-Bknockdown-induced phenotype in OS.

\section{Conclusion}

As shown in the Fig. 7, the findings indicated that silencing of Aurora-B stimulate autophagy via decreasing mTOR/ULK1 signaling pathway and result in inhibiting OS metastasis, this Aurora-B/mTOR/ULK1 axis may serve as a potential therapeutic target and prognostic marker for OS.

(See figure on next page.)

Fig. 6 Aurora-B knockdown inhibits metastasis via mTOR-mediated autophagy in vivo. 143B cells stably expressing firefly luciferase were inoculated into the tibia of female BALB/C nude mice $(n=6)$ to establish an orthotopic model of spontaneous OS $(\mathbf{a})$. After 6 weeks, mice were dissected and luminescence signals were detected $(\mathbf{b})$. Then, the number of mice with metastases was counted (c). Representative H\&E staining sections of the lungs were visualized by microscopy (d). Scale bars: $1000 \mu \mathrm{m}$ (left images) and $400 \mu \mathrm{m}$ (right images) 


\section{a}

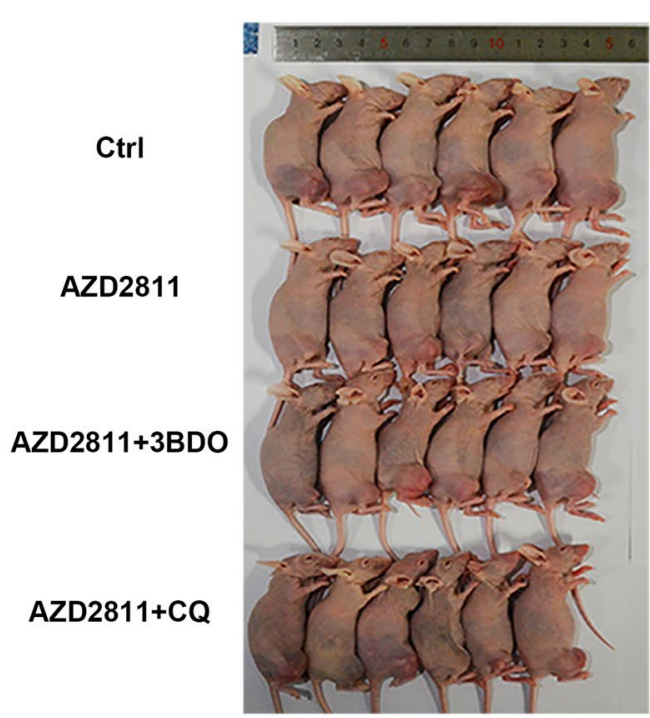

b

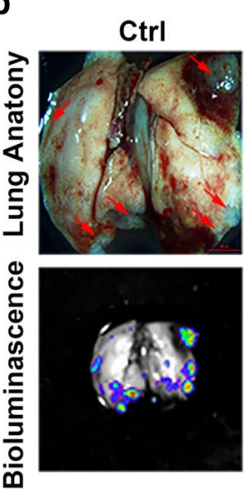

AZD2811
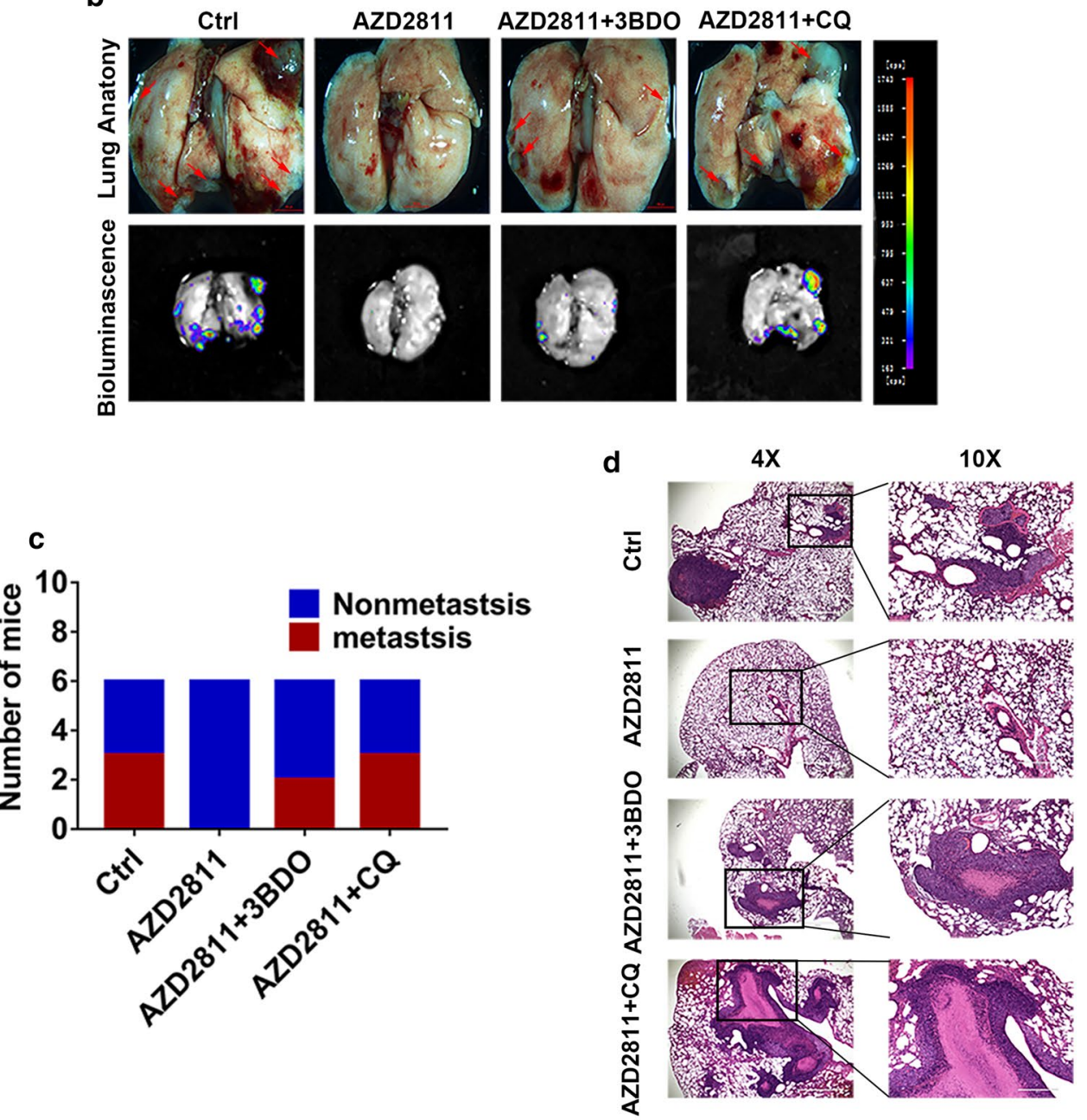


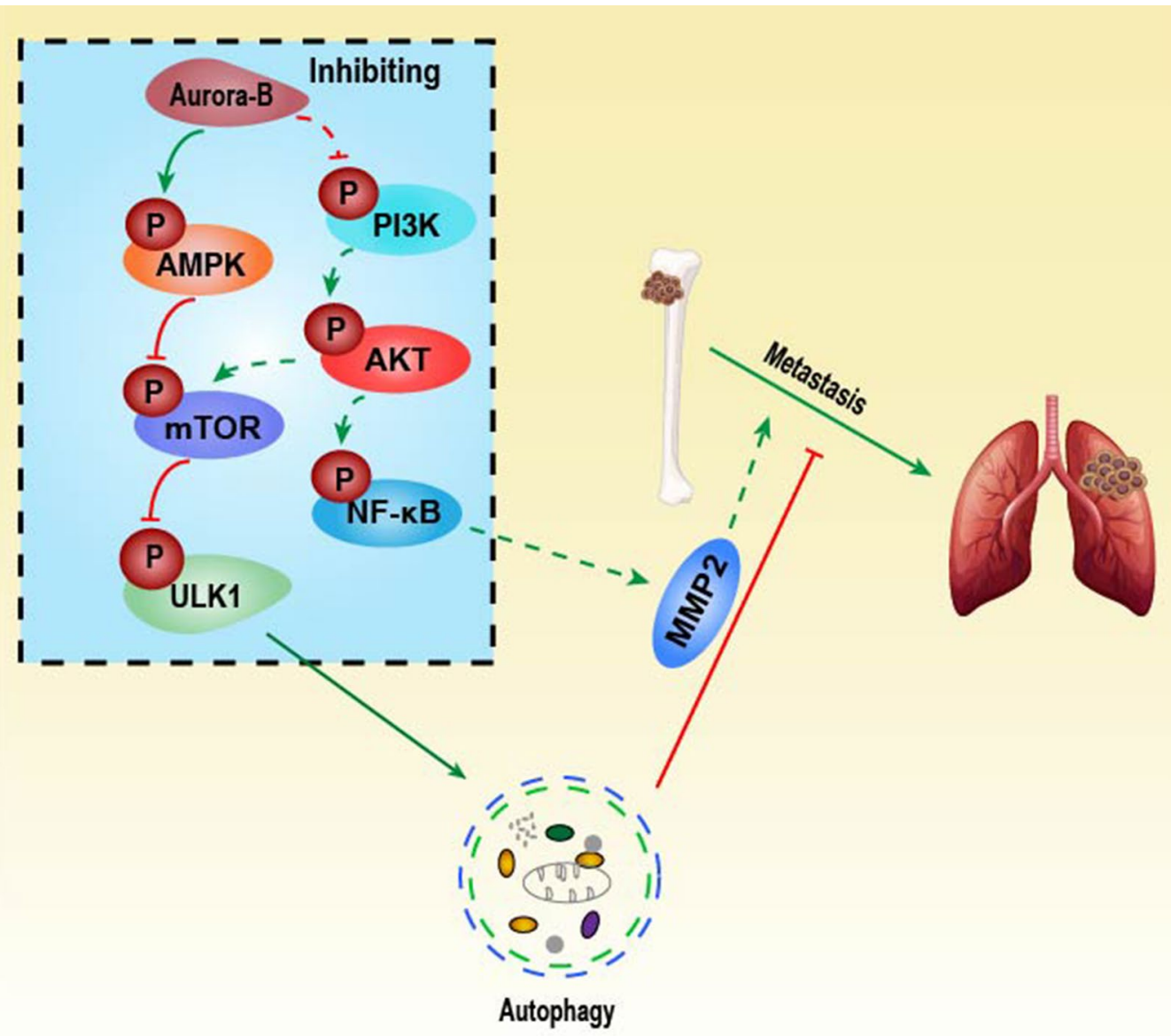

Fig. 7 Schematic model of the mechanism by which Aurora-B silencing induces autophagy to suppress metastasis in OS

\section{Abbreviations}

Aurora-B: Aurora kinase B; OS: Osteosarcoma; shRNA: Short hairpin RNA; IHC: Immunohistochemistry; GPCR: Quantitative PCR; FBS: Fetal bovine serum; $\mathrm{MOI}$ Multiplicity of Infection; HCA: Hierarchical cluster analysis; TEM: Transmission electron microscopy; CQ: Chloroquine diphosphate; EMT: Epithelial-mesenchymal transition.

\section{Acknowledgements}

Thank for the Transgenic Mouse Facility in the Institute of Translational Medicine of Nanchang University provides us with an experimental environment.

\section{Authors' contributions}

$Z L$ and $J L$ were designed this study. XW were responsible for experiments and analyzed the data. XW and JL wrote the paper. All authors collaborated to interpret results and develop the manuscript. All authors read and approved the final manuscript.

\section{Funding}

This study was supported by The National Natural Science Foundation of China (81660442, 81860472), and Natural Science Foundation of Jiangxi Province (20192ACBL21041).

\section{Availability of data and materials}

Please contact corresponding author for data requests.
Ethics approval and consent to participate

This study involving human OS tissue samples were approved by the ethics committee of The First Affiliated Hospital of Nanchang University (China) (No. Y2019-126). Informed consent from the patients was obtained in all cases. The protocols for the animal experiment were approved by The First Affiliated Hospital of Nanchang University Institutional Animal Care and Use Committee (No. IACUC-1912015).

\section{Consent for publication}

All listed authors have actively participated in the study and have read and approved the submitted manuscript.

\section{Competing interests}

The authors declare that they have no competing interests.

\section{Author details}

${ }^{1}$ Department of Orthopedic Surgery, The First Affiliated Hospital of Nanchang University, No.17 Yong Wai Zheng Street, Donghu District, Nanchang, Jiangxi 330006, People's Republic of China. ${ }^{2}$ Spine \& Spinal Cord Institute, Nanchang University, No.17 Yong Wai Zheng Street, Donghu District, Nanchang, Jiangxi 330006, People's Republic of China.

Received: 14 August 2020 Accepted: 23 November 2020

Published online: 30 November 2020 


\section{References:}

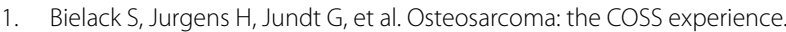
Cancer Treat Res. 2009;152:289-308.

2. Ritter J, Bielack SS. Osteosarcoma. Ann Oncol. 2010;21(Suppl 7):vii320-325.

3. Mirabello L, Troisi RJ, Savage SA. Osteosarcoma incidence and survival rates from 1973 to 2004: data from the surveillance, epidemiology, and end results program. Cancer. 2009:115:1531-43.

4. Allison DC, Carney SC, Ahlmann ER, et al. A meta-analysis of osteosarcoma outcomes in the modern medical era. Sarcoma. 2012:2012:704872.

5. Luetke A, Meyers PA, Lewis I, Juergens H. Osteosarcoma treatment - where do we stand? A state of the art review. Cancer Treat Rev. 2014:40:523-32

6. Amaravadi R, Kimmelman AC, White E. Recent insights into the function of autophagy in cancer. Genes Dev. 2016;30:1913-30.

7. White E. Deconvoluting the context-dependent role for autophagy in cancer. Nat Rev Cancer. 2012:12:401-10.

8. Wang Z, Li C, Jiang M, Chen J, Yang M, Pu J. Filamin A (FLNA) regulates autophagy of bladder carcinoma cell and affects its proliferation, invasion and metastasis. Int Urol Nephrol. 2018;50:263-73.

9. Li Z, Dong H, Li M, et al. Honokiol induces autophagy and apoptosis of osteosarcoma through PI3K/Akt/mTOR signaling pathway. Mol Med Rep. 2018;17:2719-23.

10. Yin Y, Tang L, Shi L. The metastasis suppressor gene KISS-1 regulates osteosarcoma apoptosis and autophagy processes. Mol Med Rep. 2017:15:1286-90.

11. Ruchaud S, Carmena M, Earnshaw WC. Chromosomal passengers: conducting cell division. Nat Rev Mol Cell Biol. 2007:8:798-812.

12. Al-Khafaji AS, Davies MP, Risk JM, et al. Aurora B expression modulates paclitaxel response in non-small cell lung cancer. Br J Cancer. 2017;116:592-9.

13. Bogen D, Wei JS, Azorsa DO, et al. Aurora B kinase is a potent and selective target in MYCN-driven neuroblastoma. Oncotarget. 2015;6:35247-62.

14. González-Loyola A, Fernández-Miranda G, Trakala M, et al. Aurora B Overexpression Causes Aneuploidy and p21Cip1Repression during Tumor Development. Mol Cell Biol. 2015;35:3566-78.

15. Huang PY, Li Y, Luo DH, et al. Expression of Aurora-B and FOXM1 predict poor survival in patients with nasopharyngeal carcinoma. Strahlenther Onkol. 2015;191:649-55.

16. Zhang $\mathrm{Y}$, Jiang $\mathrm{C}$, Li H, et al. Elevated Aurora B expression contributes to chemoresistance and poor prognosis in breast cancer. Int J Clin Exp Pathol. 2015;8:751-7.

17. Takeshita M, Koga T, Takayama K, et al. Aurora-B overexpression is correlated with aneuploidy and poor prognosis in non-small cell lung cancer. Lung Cancer. 2013;80:85-90.

18. Bertran-Alamillo J, Cattan V, Schoumacher M, et al. AURKB as a target in non-small cell lung cancer with acquired resistance to anti-EGFR therapy. Nat Commun. 2019;10:1812.

19. He JY, Xi WH, Zhu LB, et al. Knockdown of Aurora-B alters osteosarcoma cell malignant phenotype via decreasing phosphorylation of VCP and NF-kappaB signaling. Tumour Biol. 2015;36:3895-902.

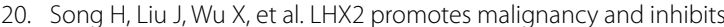
autophagy via mTOR in osteosarcoma and is negatively regulated by miR-129-5p. Aging (Albany NY). 2019·11:9794-810.

21. Jung $\mathrm{CH}, \mathrm{Ro} \mathrm{SH}$, Cao J, Otto NM, Kim DH. mTOR regulation of autophagy. FEBS Lett. 2010;584:1287-95.

22. Rabanal-Ruiz Y, Otten EG, Korolchuk VI. mTORC1 as the main gateway to autophagy. Essays Biochem. 2017:61:565-84

23. Wirth $M$, Joachim J, Tooze SA. Autophagosome formation-the role of ULK1 and Beclin1-PI3KC3 complexes in setting the stage. Semin Cancer Biol. 2013;23:301-9.
24. Cao C, Huang W, Zhang N, et al. Narciclasine induces autophagy-dependent apoptosis in triple-negative breast cancer cells by regulating the AMPK-ULK1 axis. Cell Prolif. 2018:51:e12518.

25. Rakhmanova $V$, Jin M, Shin J. Inhibition of mast cell function and proliferation by mTOR activator MHY1485. Immune Netw. 2018;18:e18.

26. Zhang M, Liu S, Chua M-S, et al. SOCS5 inhibition induces autophagy to impair metastasis in hepatocellular carcinoma cells via the PI3K/Akt/ mTOR pathway. Cell Death Disease . 2019a;10:799.

27. Zhao GS, Gao ZR, Zhang Q, et al. TSSC3 promotes autophagy via inactivating the Src-mediated PI3K/Akt/mTOR pathway to suppress tumorigenesis and metastasis in osteosarcoma, and predicts a favorable prognosis. J Exp Clin Cancer Res. 2018;37:188.

28. Zhao Z, Jin G, Yao K, et al. Aurora B kinase as a novel molecular target for inhibition the growth of osteosarcoma. Mol Carcinog. 2019;58:1056-67.

29. Chiou SK, Hoa N, Hodges A, Ge L, Jadus MR. Indomethacin promotes apoptosis in gastric cancer cells through concomitant degradation of Survivin and Aurora B kinase proteins. Apoptosis. 2014;19:1378-88.

30. Schecher $\mathrm{S}$, Walter $\mathrm{B}$, Falkenstein $\mathrm{M}$, et al. Cyclin $\mathrm{K}$ dependent regulation of Aurora B affects apoptosis and proliferation by induction of mitotic catastrophe in prostate cancer. Int J Cancer. 2017;141:1643-53.

31. Zhu X, Liu Z, Peng A, et al. Inhibition of Aurora-B suppresses osteosarcoma cell migration and invasion. Exp Ther Med. 2014;7:560-4.

32. Yu JJ, Pi WS, Cao Y, et al. Let-7a inhibits osteosarcoma cell growth and lung metastasis by targeting Aurora-B. Cancer Manag Res. 2018:10:6305-15.

33. Niu J, Yan T, Guo W, Wang W, Zhao Z. Insight into the role of autophagy in osteosarcoma and its therapeutic implication. Front Oncol. 2019;9:1232.

34. Choi KS. Autophagy and cancer. Exp Mol Med. 2012;44:109-20.

35. Zhang M, Liu S, Chua M-S, et al. SOCS5 inhibition induces autophagy to impair metastasis in hepatocellular carcinoma cells via the PI3K/Akt/ mTOR pathway. Cell Death Dis. 2019b;10:612.

36. Liu K, Yu Q, Li H, et al. BIRC7 promotes epithelial-mesenchymal transition and metastasis in papillary thyroid carcinoma through restraining autophagy. Am J Cancer Res. 2020;10:78-94.

37. Liu W, Jiang D, Gong F, et al. miR-210-5p promotes epithelial-mesenchymal transition by inhibiting PIK3R5 thereby activating oncogenic autophagy in osteosarcoma cells. Cell Death Dis. 2020;11:93.

38. Klionsky D, Abdelmohsen K, Abe A, et al. Guidelines for the use and interpretation of assays for monitoring autophagy (3rd edition). Autophagy. 2016;12:1-222

39. Torres-Quiroz F, Filteau M, Landry CR. Feedback regulation between autophagy and PKA. Autophagy. 2015;11:1181-3.

40. Parzych KR, Klionsky DJ. An overview of autophagy: morphology, mechanism, and regulation. Antioxid Redox Signal. 2014;20:460-73.

41. Tamargo-Gomez I, Marino G. AMPK: regulation of metabolic dynamics in the context of autophagy. Int J Mol Sci. 2018;19:3812.

42. Zhang Y, Fan Y, Huang S, et al. Thymoquinone inhibits the metastasis of renal cell cancer cells by inducing autophagy via AMPK/mTOR signaling pathway. Cancer Sci. 2018;109:3865-73.

43. Zhu LB, Jiang J, Zhu XP, et al. Knockdown of Aurora-B inhibits osteosarcoma cell invasion and migration via modulating PI3K/Akt/NF-kappaB signaling pathway. Int J Clin Exp Pathol. 2014;7:3984-91.

\section{Publisher's Note}

Springer Nature remains neutral with regard to jurisdictional claims in published maps and institutional affiliations. 